\title{
Necesidades socioeconómicas y medioambientales de la población de la Zona Oriental de El Salvador, primera etapa: \\ Departamento de San Miguel.
}

Vilma Evelyn Gómez Zetino

Máster en Docencia Universitaria

Coordinadora de Investigación, Universidad de Oriente

Email: zetinosv@yahoo.es

\section{RESUMEN}

Recepción: 2012-06-12

Aceptación: 2012-08-16

El conocimiento de las diferentes variables socioeconómicas, medioambientales y su vinculación con la región debe ser considerado como el punto de partida para desarrollar propuestas de proyectos, relacionados con las tres funciones básicas de la Educación Superior, cuyo último fin sea el desarrollo local.

Ante todo, es significativo destacar que, la Zona Oriental de El Salvador presenta una serie de características que propician que la magnitud y desarrollo de los fenómenos alcancen enfoques variados, entre los departamentos y municipios que la conforman. Entre las variables que afecta las condiciones de vida de la población, poniendo en peligro el desarrollo sostenible de la zona, se destacan la migración, el bajo desarrollo industrial, la delincuencia, la situación precaria ambiental y la baja productividad agrícola.

En lo concerniente al mapeo de la problemática del territorio, esto se puede lograr a través del desarrollo de un diagnóstico participativo, identificando, priorizando y proponiendo alternativas de solución a sus necesidades y dificultades. Esto con la finalidad de involucrar a todos los actores vinculados a la zona, como los referentes claves para obtener la información, de manera que se transformen en entes participativos que construyen su propio desarrollo.

Palabras claves: diagnóstico, necesidades socioeconómicas, necesidades medioambientales, desarrollo local y territorial. 
[ 86 ] Universidad Católica de El Salvador.

\section{ABSTRACT}

Knowledge of the socioeconomic, environmental variables and their link to the region must be considered as a starting point to develop project proposals, related to the three basic functions of Higher Education, with the ultimate goal of local development.

Above all, it is meaningful to emphasize that the Eastern part of El Salvador has certain characteristics that allow that magnitude of the phenomena to take different approaches within the municipalities that belong to it. Among the variables that affect the living conditions of the population, raising the sustainable development of the area, we can mention: migration, low industrial development, delinquency, precarious environmental situation and low agricultural productivity.

Regarding the mapping of the land problem, it can be achieved through the development of a participative diagnosis, identifying, prioritizing and proposing alternatives of solution to their needs and difficulties. This is with the aim of including all the stakeholders in the area, as the key reference for information and become into beings that can build their own development.

Key words: diagnosis, socioeconomic needs, environmental needs, local and territorial development.

\section{INTRODUCCIÓN}

El departamento de San Miguel se encuentra ubicado en la Zona Oriental de El Salvador. Su territorio alcanza 2, 077,1 kilómetros cuadrados, tiene una población de 434,003 personas, con una densidad poblacional de 209 habitantes por $\mathrm{Km}^{2}$, lo que lo hace el quinto departamento más poblado de El Salvador.
La distribución por género dentro del departamento señala que existen 232,328 mujeres y 201,675 hombres, los que representan un índice de masculinidad del 87\%; es decir 87 hombres por cada 100 mujeres. Esto se considera vinculado a las corrientes migratorias provenientes de las áreas rurales que son mayores para el género femenino, debido a la posibilidad de insertarse en la actividad eco-

Volumen No. 1 Diagnóstico sobre las necesidades socioeconómicas y medioambientales de la población de la Zona Oriental de El Salvador. Primera etapa diagnóstico del departamento de San Miguel. 
nómica de forma más rápida. Es importante mencionar que la población en este departamento se distribuye casi de manera equitativa entre el área urbana (50.6) y rural (49.4)1.

Administrativamente, el departamento de San Miguel posee 20 municipios, siendo el más poblado de ellos el municipio que lleva su mismo nombre. En cuanto a la condición familiar, las estadísticas muestran que en el departamento existe un alto porcentaje de familias integradas por 3 ó 4 miembros. Entre los bienes y servicios que los pobladores poseen con más frecuencia están los que se citan a continuación: Televisor, teléfono fijo y celular, refrigeradora, equipo de sonido y servicio de televisión por cable.

Respecto al ámbito educativo existen las modalidades públicas y privadas, teniendo predominio la primera. Con respecto a la Educación Superior, en este departamento existen siete instituciones de este tipo.

En cuanto a la atención de salud, el departamento oriental cuenta tanto con servicios privados como públicos, en donde predominan éstos últimos por brindarse a nivel hospitalario y en clínicas asistenciales. Es así, a pesar de que en la cabecera del municipio de San Miguel se encuentran tres importantes cen-

1 Ministerio de Economía, Dirección General de Estadísticas y Censos.2008. VI Censo de población y V de vivienda 2007 tros privados de salud, que aportan significativamente a la economía del departamento.

En relación con la infraestructura, el departamento de San Miguel ha evolucionado. Actualmente cuenta con una red vial que presenta mejoras significativas. Sin embargo, todavía existen una serie de problemas que no logran superarse.

En cuanto a la vivienda y otro tipo de infraestructura vinculada a los servicios con que cuenta cada municipio, se destaca la presencia de vivienda mixta con materiales de construcción modernos, quedando muy pocas que utilizan materiales autóctonos. Situación similar han seguido los edificios destinados a prestar servicios y que, de alguna manera, están vinculados con el desarrollo de los diferentes municipios, tales como clínicas, hospitales, escuelas, alcaldías, mercados y parques por mencionar los más sobresalientes. Esto desemboca que se conserve muy poco de las edificaciones propias de cada región.

Si se toma como referencia la definición de medio ambiente manejada por la Conferencia de las Naciones Unidas, se puede decir que, "el conjunto de componentes físicos, químicos, biológicos y sociales capaces de causar efectos directos o indirectos, en un plazo corto o largo, sobre los seres vivos y las actividades 
humanas" ha cambiado de manera drástica y acelerada. Esto ha impactado directamente en las condiciones de salud, en la producción de alimento, en el clima, el agua y en el suelo de todo el departamento de San Miguel.

Hay que hacer notar que, en los últimos años el departamento de San Miguel ha visto incrementadas sus condiciones de vulnerabilidad ante fenómenos climáticos, aumentando las condiciones de riesgo, que repercuten en la economía y la calidad de vida de la población, tanto en el campo como en la ciudad.

Es importante mencionar que a nivel departamental, la valoración que la población hace de los recursos naturales que se encuentran disponibles para la satisfacción de sus necesidades, desde un enfoque de desarrollo sostenible, es incipiente.

\section{MÉTODO}

\subsection{Participantes}

La muestra considerada en el diagnóstico estuvo integrada por 1,149 habitantes de diferentes municipios del departamento de San Miguel. A ellos se les agrupó en dos categorías: Zona norte (Carolina, Ciudad Barrios, Chapeltique, Nuevo Edén de San Juan, San Antonio del Mosco, San Gerardo, San Luis de la Reina, Sesori), centro (San Miguel, Comarcarán, Chinameca, Lolotique, Moncagua, Nueva Guadalupe, Quelepa, Uluazapa) y Zona sur (Chirilagua, El Tránsito, San Jorge, San Rafael Oriente).

\subsection{Instrumento utilizado}

A través de la definición de indicadores para cada una de las variables, consideradas en el estudio, se diseñó un cuestionario con preguntas de respuestas múltiples. Cada una de las preguntas ofrecía un listado de opciones que podían seleccionarse según se consideraran pertinentes. Para este tipo de preguntas, el número de respuestas fue delimitado por el número de opciones que el investigador incluyó en el listado.

\subsection{Procedimiento}

El levantamiento y análisis de la información dio inicio con la zonificación del departamento, cálculo de la muestra (una por zona) y el diseño del instrumento. Para la aplicación del instrumento se contó con un equipo de cinco personas, capacitadas de manera previa, para que dieran las mismas instrucciones a todos los participantes; asegurándoles que sus repuestas serían tratadas de forma anónima, confidencial y voluntaria.

Volumen No. 1 Diagnóstico sobre las necesidades socioeconómicas y medioambientales de la población de la Zona Oriental de El Salvador. Primera etapa diagnóstico del departamento de San Miguel. 


\section{RESULTADOS}

En el análisis del panorama del departamento de San Miguel se incorporaron variables sociodemográficas, además de otras de carácter puntual como la estructura familiar y económica, vivienda, educación, salud, infraestructura y medioambiente. A través de todas ellas se buscó tener un panorama holístico de la situación del territorio.

Mediante las variables sociodemográficas se tuvo una idea más objetiva de la población que formó parte del estudio. Concretamente, en relación al género, se contó con homogeneidad dentro de la muestra. Ésta fue tipificada en las categorías de adultos jóvenes, adultos y adultos mayores, los cuales fueron seleccionados al azar, de entre los veinte municipios que conforman el departamento de San Miguel.

\subsection{Situación socioeconómica}

\section{a. Estructura familiar y económica}

Para valorar esta variable se consideraron los indicadores de ocupación, tiempo laboral, sector de empleo, ingresos, actividades productivas, transporte, canasta básica alimentaria, servicios básicos, problemas comunitarios y migración.
Un alto porcentaje de la población del departamento subsiste a través de un empleo, generado en un $36 \%$ por la empresa privada y en un $29 \%$ por instituciones estatales. Dentro de la investigación también se determinó que el nivel de ingreso, de más del $50 \%$ de la población, oscila entre $\$ 200$ a $\$ 800$ dólares.

Pero también, se encontró que la economía de la familia es complementada con el aporte de actividades no formales y con remesas, pues de los consultados, el 70\% de ellos afirmó tener parientes en el extranjero.

A pesar de estos insumos, el alto costo de la vida hace insuficiente estos ingresos para la satisfacción de las necesidades básicas de la familia.

Los resultados señalan que en el departamento, la actividad productiva es mínima. Esto permite deducir que se vive en una potencial sociedad de consumo, cuyo interés no se fundamenta en el desarrollo del sector producti-

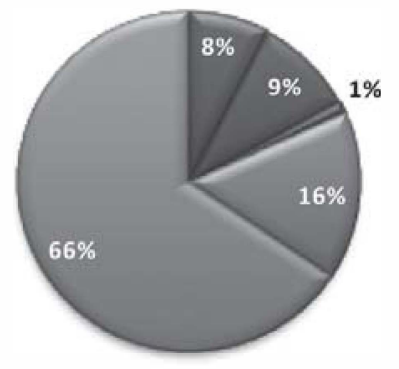

$8 \%$ NO RESPONDIERON

9\% GANADERIA

$1 \%$ FORESTAL

16\% AGRICULTURA

66\% OTRASACTNIDADES 
vo. Tal afirmación es palpable en los datos obtenidos a través de los indicadores ocupación $\mathrm{y}$ tipos de ingresos percibidos por la familia.

En cuanto a la cobertura de la canasta básica, solo el $35 \%$ de la población afirmó cubrir esta necesidad en su totalidad; mientras que el $52 \%$ de los consultados señalan que esta necesidad es cubierta en parte. Por lo tanto, el 13\% de la población restante, no cubre ni total ni parcialmente esta necesidad.

Para el analista económico, Ricardo Perdomo, el salario mínimo rural está por debajo del precio de la canasta básica alimentaria, por lo que únicamente se alcanza a cubrir un $74 \%^{2}$ de ella. Esto da como resultado un claro desajuste entre los ingresos económicos de la población y los costos de la alimentación. De entre todos los consultados, solamente el $1 \%$ aseguró tener una alimentacion que podría clasificarse como balanceada.

Otro de los puntos de interés vinculados a la economía familiar es el tipo y uso de la energía, el cual en las familias salva

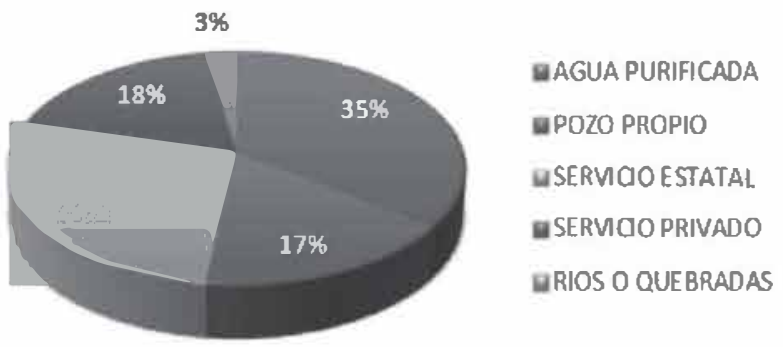

Figura 2. Procedencia del agua para consumo

Volumen No. 1 Diagnóstico sobre las necesidades socioeconómicas y medioambientales de la población de la Zona Oriental de El Salvador. Primera etapa diagnóstico del departamento de San Miguel. 
En el ámbito global, la sociedad salvadoreña carga con un sin número de problemas, unos más complejos que otros, haciendo que tanto la vida individual como la convivencia colectiva sean sumamente difíciles. Los consultados hacen referencia que entre los problemas que más afectan el desarrollo en la actualidad son el accionar de maras (30\%) y la falta de empleo (21\%), principalmente. A estos le siguen la pobreza (17\%) y la delincuencia (14\%). A juicio de un $11 \%$ de los encuestados existen otra serie de problemas no específicas, mientras que para el 7\% de ellos el origen es multicausal.

Sobre este mismo tema, en el ámbito local (municipal), se buscó indagar sobre los problemas vinculados a la familia, destacándose situaciones como desintegración familiar, maltrato infantil, violencia entre parejas, drogadicción y por último las violaciones sexuales. Las respuestas reflejan que se tiene una crisis al interior de muchas de las familias migueleñas, por lo que es trascendental el desarrollo de programas y proyectos que aborden esta problemática.

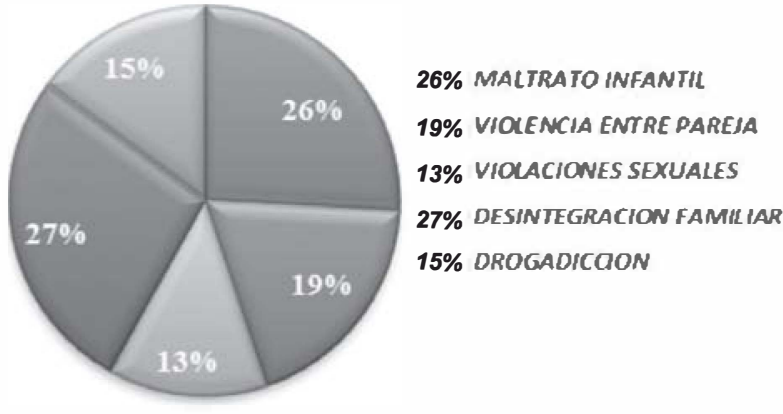

Figura 3. Problemas frecuentes en las comunidades

\section{b. Vivienda}

Más del 30\% de déficit cualitativo a nivel nacional se encuentra en los departamentos de Sonsonate (11.04\%), Ahuachapán (10.41\%) y San Miguel $(10.08 \%)^{3}$. De aquí se parte para asegurar que en el departamento de San Miguel, la situación habitacional de la población se relaciona fundamentalmente con aspectos de carácter cualitativo.

En la consulta, el 72\% de la muestra expresó tener casa propia. Sin embargo, éstas no reunen las condiciones de seguridad y comodidad necesarias, pues al preguntar sobre el tipo de construcción, solamente el 35 \% dijo tener una vivienda de tipo mixta. Además, existe una amplia brecha relacionada con los servicios básicos que poseen las viviendas. Para el caso, del 100\% de los participantes, solamente el 35\% de ellos manifestó poseer el servicio de energía eléctrica en sus hogares; mientras que un $19 \%$ dijo contar con cañerias de aguas negras y un $17 \%$ aseguró tener servicio de agua potable.

\section{c. Educación}

En este contexto, los resultados señalan que existe un evidente estancamiento. El 13\% de los consultados sólo ha cursado hasta tercer

3 FUNDASAL, Anteproyecto de Ley de Vivienda de Interés Social: Una necesidad palpable. Una propuesta para el acceso de los más pobres. 


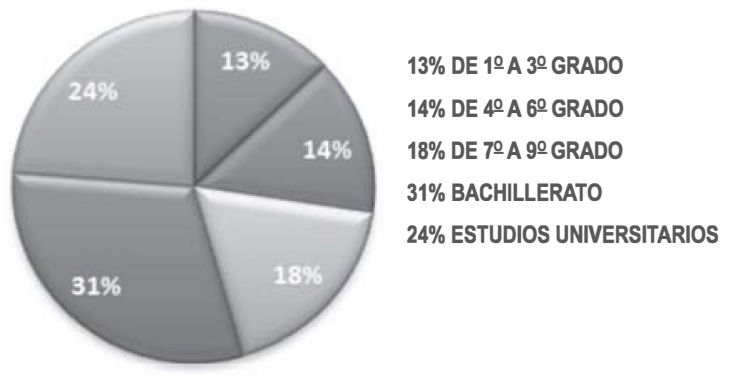

Figura 4. Escolaridad en el departamento de San Miguel

grado de Educación Básica, mientras que un $24 \%$ sí ha accedido a estudios universitarios. Esto da pauta para establecer que existe una brecha de diferenciación muy marcada a nivel educativo. Resultados similares pueden ser comparados con lo reportado en el Censo de Población del año 2007.

Al realizar un análisis de la problemática de la escuela, el ausentismo (28\%), reprobación (22\%), repitencia (21\%) y deserción (20\%) son los motivos más comunes. Esto enfatiza la urgencia de realizar acciones que avancen de manera significativa y pertinente con respecto a la calidad de la educación.

\section{d. Salud}

El sistema de salud en El Salvador es y ha sido un tema de fuertes críticas y reflexiones dentro de todo nivel social. El sistema de salud no logra cumplir las expectativas, bajo la perspectiva de aplicación de modelos curativos y no de modelos preventivos, en el abor- daje de los problemas de salud que enfrenta la población. Esto se refleja en la presistencia de un alto número de casos de enfermedades respiratorias y gastrointestinales, padecidas con frecuencia por grupos vulnerables (niños y adultos mayores). Estas enfermedades, en la mayoría de los casos, están asociadas a una higiene personal y alimenticia deficiente.

Es importante mencionar que para el tratamiento de estas enfermedades se invierte gran parte del presupuesto destinado a los centros de salud pública, que son visitados por un $59 \%$ de los consultados en este estudio. Lo anterior denota la importancia que tiene la promoción de programas preventivos integrales en el tratamiento del proceso salud/enfermedad, a fin de propiciar la toma de conciencia por parte de la población, en cuanto al beneficio tanto personal como social. A su vez, esto fortalece la eficiencia y eficacia de los servicios ofrecidos.

\section{e. Infraestructura}

En relación a este tema se abordaron dos puntos básicos: red vial e infraestructura básica. En cuanto al primer punto, los entrevistados opinan que existe un deterioro en las vías de comunicación del departamento (52\%). Los encuestados también señalan que la disponibilidad de infraestructura por municipio no es completa. Dicha opinión es compartida

Volumen No. 1 Diagnóstico sobre las necesidades socioeconómicas y medioambientales de la población de la Zona Oriental de El Salvador. Primera etapa diagnóstico del departamento de San Miguel. 
por el $99 \%$ de los consultados. De aquí que es importante que los gobiernos locales gestionen proyectos que permitan contar con todos los recursos que sean necesarios para potenciar el desarrollo local y el bienestar de la población.

\subsection{Situación medioambiental}

Cada uno de los lugares del planeta en donde la vida es posible, reúnen características que lo convierten en territorios únicos. Así, al consultar a la población que habita en los municipios del departamento de San Miguel sobre las condiciones ambientales que sus municipios ofrecen, el $36 \%$ de ellos respondió que su riqueza medio ambiental está fundamentada en los recursos naturales que poseen. Mismos que deberían estar encaminados a conservar la salud y la calidad de vida de los pobladores, pero cuya situación en la actualidad es regular.

La opinión del $61 \%$ de los consultados coincide que el medio ambiente tiene importancia en la salud. Esta opinión es respaldada por la Organización Mundial para la Salud (OMS), como la percepción que las personas tienen de su lugar en la vida, en un contexto determinado y un sistema de valores, en relación con sus propios objetivos y expectativas. Esto involucra también la salud física, el estado psicológico, el nivel de independencia, las re- laciones sociales y las relaciones con su ambiente.

Los problemas ambientales que más preocupan a la población del departamento de San Miguel son la deforestación y la contaminación atmosférica. En torno al tema, la percepción de la población ante este cuestionamiento es que, los gobiernos locales aportan poco al respecto, lo cual es verificable en la carencia de políticas o, en otros casos, en la débil aplicación de la legislación existente. También es importante analizar que muchos de los problemas de salud que sufre la población se encuentran estrechamente vinculados a las condiciones que presentan los recursos naturales que integran los ecosistemas, entre los que se encuentran el agua, el aire, el suelo, la fauna y la flora.

Otra de las grandes dificultades, en torno al mismo tema, es el limitado conocimiento que tiene la población en materia de legislación ambiental. Al consultar a la población en re-

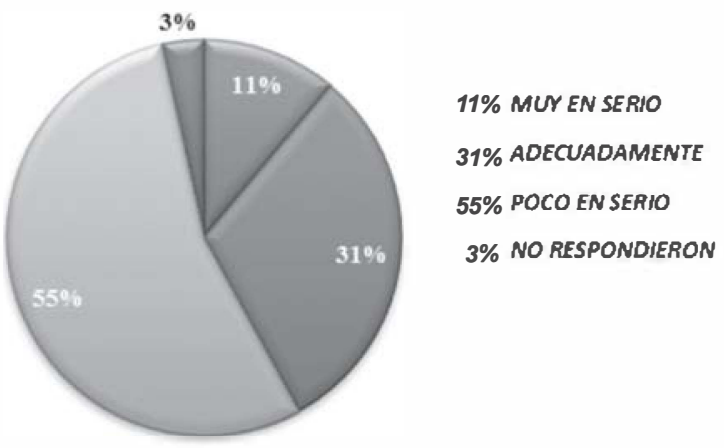

Figura 5. Interés de los gobiernos locales en problemas ambientales 
lación a este punto, el $48 \%$ dijo desconocer del tema y solamente el $24 \%$ de ellos afirmó conocer un poco. Lo anterior señala la necesidad de realizar proyectos de divulgación y/o capacitación en materia ambiental. Como resultado de esta limitación, la cultura de la denuncia es un tema pendiente de resolver en el departamento de San Miguel y en El Salvador.

Sin embargo, en los últimos años han existido ejemplos en donde la población, de manera clara y contundente, ha expresado su descontento respecto a algunas situaciones. Entre los casos más recientes figuran la Construcción de la presa el Chaparral, la contaminación por plomo en Sitio El Niño, la planta de carbón en La Unión, etc. También es importante destacar que estas iniciativas se han concretado como resultado de la participación de instituciones que velan por los derechos humanos, demostrando que la población no cuenta con las herramientas organizativas para enfrentar este tipo de problemas.

Por otra parte, en algunos municipios han dado pasos de mejora en este aspecto, pues al menos un $74 \%$ de los consultados afirman que en su municipio cuentan con un tren de aseo. Sin embargo todavía se realizan prácticas no amigables con el ambiente como quemar basura y tirarla a la intemperie. Por su parte, un $29 \%$ de los entrevistados asegura estar reciclando la basura. Este dato es un buen indicio de cambio, mientras que un 7\% de ellos la entierra. Aunque no es lo mejor, el impacto ambiental que genera es menor. El panorama es caótico pero reversible, seguido de la participación ciudadana, guiada a través de políticas claras de desarrollo.

\section{CONCLUSIONES}

El diagnóstico realizado en el departamento de San Miguel presenta un panorama holístico de las siguientes variables: estructura familiar y económica, vivienda, educación, salud, infraestructura y medioambiente.

Los resultados obtenidos presentan en un primer momento una sociedad sumergida en una grave crisis. Sin embargo, es importante mencionar que en torno a ésta, el gobierno y los gobiernos locales del departamento están realizando esfuerzos, aunque no satisfacen completamente a la población, debido a que éstos no responden a planes y programas diseñados sobre la base de políticas claras que impulsen el desarrollo del país. Por ende, dentro de cada municipio existe un marcado divorcio entre estos sujetos. Por otra parte, las instituciones no gubernamentales, la cooperación internacional y la sociedad civil también tienen una lectura del entorno, realizando sus propios esfuerzos, los cuales se vuelven dispersos debido a la falta del consenso entre los involucrados.

Volumen No. 1 Diagnóstico sobre las necesidades socioeconómicas y medioambientales de la población de la Zona Oriental de El Salvador. Primera etapa diagnóstico del departamento de San Miguel. 
En definitiva, los problemas son diversos, dejando en evidencia un sistema económico frágil, una limitada satisfacción de las necesidades básicas de la población, una crisis social creciente de alta peligrosidad por el nivel de organización que poseen y servicios básicos deficientes e insuficientes. Todo esto sumado a una educación vulnerable, que no responde al ritmo globalizado del mundo, que refleja de manera clara y contundente su retraso en todos los sentidos; con servicios de salud exiguos, una deuda infraestructural tanto de índole cualitativo como cuantitativa $y$, finalmente, un medio ambiente que pone en riesgo no solamente su subsistencia sino también la vida misma.

Lo anterior es resultado de un manejo irresponsable de los recursos naturales disponibles en la región, sellado con un alto nivel de conformismo y acomodamiento por parte de la sociedad que observa de manera inmutable cada uno de los fenómenos que acontecen a su alrededor.

\section{REFERENCIAS BIBLIOGRÁFICAS}

Banco Central de Reserva. Características de los remitentes de remesas familiares desde Estados Unidos. De, García Xiomara, De, Palacios Morena.

Canasta básica rural incrementa 35\% en un año. La Prensa Gráfica, El Salvador, 29 de junio de 2011.Economia.

Cámara de Comercio e Industria de El Salvador, 2010. Propuestas para el Combate Integral de la Delincuencia en El Salvador.

Classen-Bauer, Ingrid (et.al). 1998. Estudio nacional sobre el Sistema de Formación Inicial de docentes de Educación Básica $1^{\circ}$ y $2^{\circ}$ ciclos. Ministerio de Educación- GTZ.

Centro Nacional de Registro, Monografía del departamento de San Miguel. Instituto Geográfico y de Catastro Nacional.

Ministerio de Economía, Dirección General de Estadísticas y Censos.2008. VI Censo de población y V de vivienda 2007.

Ministerio de Salud. Informe de Labores Ministerio de Salud 2009-2010. República de El Salvador. 